\title{
Slow Down and Concentrate: Time for a Paradigm Shift in Fall Prevention among People with Parkinson's Disease?
}

\author{
Emma L. Stack and Helen C. Roberts \\ Academic Geriatric Medicine, Faculty of Medicine, University of Southampton, Mailpoint 807, \\ University Hospital Southampton, Tremona Road, Southampton SO16 6YD, UK \\ Correspondence should be addressed to Emma L.Stack; els1n09@soton.ac.uk
}

Received 4 October 2012; Revised 10 December 2012; Accepted 17 January 2013

Academic Editor: Margaret KY Mak

Copyright @ 2013 E. L. Stack and H. C. Roberts. This is an open access article distributed under the Creative Commons Attribution License, which permits unrestricted use, distribution, and reproduction in any medium, provided the original work is properly cited.

Introduction. We know little about how environmental challenges beyond home exacerbate difficulty moving, leading to falls among people with Parkinson's (PwP). Aims. To survey falls beyond home, identifying challenges amenable to behaviour change. Methods. We distributed 380 questionnaires to PwP in Southern England, asking participants to count and describe falls beyond home in the previous 12 months. Results. Among 255 responses, 136 PwP (diagnosed a median 8 years) reported falling beyond home. They described 249 falls in detail, commonly falling forward after tripping in streets. Single fallers (one fall in 12 months) commonly missed their footing, walking, or changing position and recovered to standing alone or with unfamiliar help. Repeat fallers (median falls, two) commonly felt shaken or embarrassed and sought medical advice. Very frequent fallers (falling at least monthly; median falls beyond home, six) commonly fell backward, in shops and after collapse but often recovered to standing alone. Conclusion. Even independently active PwP who do not fall at home may fall beyond home, often after tripping. Falling beyond home may result in psychological and/or physical trauma (embarrassment if observed by strangers and/or injury if falling backwards onto a hard surface). Prevention requires vigilance and preparedness: slowing down and concentrating on a single task might effectively prevent falling.

\section{Introduction}

Postural instability is common in Parkinson's disease (PD): falls are likely to be a frequent problem for most people with Parkinson's (PwP) by 10 years after diagnosis [1]. Approximately one third of elderly people falls in any given year but approximately two thirds of PwP [2]. Most falls among PwP happen at home; for example, $80 \%$ of the 639 fall recorded over six months in one fall-prevention trial [3]. Current opinion about preventing falls in PD favours a multimodal approach: a combination of exercise and developing new movement strategies, coupled with optimal medical management [4].

$\mathrm{PwP}$ use attentional mechanisms and other cues to compensate for movement difficulty [5-7]: it is possible that they would find a distracting environment more challenging than one in which they could preserve focus. Small, qualitative studies have explored this possibility in relationship to walking, though not falls. As Lamont et al. wrote, after discussing community walking in focus groups with 18 PwP, "Challenging environments that demand attention may compromise the ability to walk" [8]. Jones et al. concluded from 20 semistructured interviews that "People with PD need to constantly assess and drive their walking performance" using "attentional resources, which can themselves be compromised" [9]. Most PwP experience progressive gait difficulties $[10,11]$ : it is possible that they would find unfamiliar environments progressively more challenging over time.

Yet we know little about the specific circumstances in which PwP fall beyond home. Li et al. described outdoor falls (three-quarters of which were "attributable to modifiable environmental factors") as a "neglected public health problem" [12]. While previous studies on "community walking" $[8,9]$ have identified factors that help or hinder walking, they have not specifically identified factors that increased or decreased stability. If PwP learn to avoid and/or otherwise 
manage the key situations in which falls tend to happen, they may prevent a number of falls.

We aimed to survey falls beyond home among PwP, identifying challenges that might be manageable through behaviour change. We proposed a postal survey to allow a wide cross-section of people to describe what had happened when they fell beyond their homes.

\section{Methods}

We constructed a simple questionnaire addressing falls beyond home, that is, not in the familiar environment of their own home or garden but including allotments (small plots of publicly owned land rented to individuals, usually for growing vegetables) and the homes and gardens of friends and family. The questionnaire containing instructions throughout was accompanied with an information sheet and a stamped addressed reply envelope for postal return. We asked the participant's age and gender, date of diagnosis, use of mobility aids indoors and out, and how many times they had fallen at home and beyond in the previous 12 months. We did not define a fall in the questionnaire, offering respondents scope to describe what they considered a fall. We intended to disregard any descriptions in which the individual (a) did not come to rest on the ground or another lower level, (b) fell out of a bed or chair asleep, or (c) was knocked to the ground. We provided space for them to answer the following questions (used previously face-to-face and via falls diaries $[13,14])$ for up to three of their most recent falls beyond home.

(1) Where did you fall?

(2) What were you doing?

(3) Why do you think you fell?

(4) How did you land?

(5) Then what happened?

Limiting repeat fallers to describing no more than three falls meant that no individual or subgroup would dominate the snapshot of falls reported. Approximately 350 questionnaires were distributed via 30 branches of Parkinson's UK across Southern England. Additionally, via one geriatrician's clinic we handed out approximately 30 questionnaires to people who reported a fall beyond home during a consultation. Recipients took the questionnaires home to consider, complete, and/or post back.

We entered all data received onto an excel spreadsheet, including all legible descriptions of falls. Using simple content analysis, we categorized and counted responses to each question. In reporting, we present totals for the whole sample, single fallers, and repeat fallers. To illustrate the circumstances in which very frequent fallers described falling, we present those who fell at least monthly separately from other repeat fallers (who fell from 2 to 11 times in 12 months).

We conducted the study with the approval of the Southampton and South West Hampshire Research Ethics Committee (B).

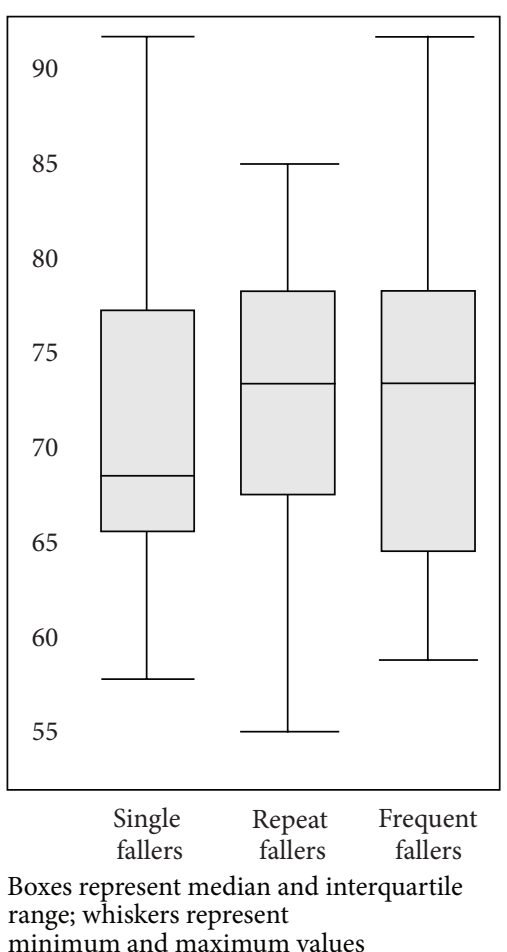

FIgUre 1: Box and Whisker Plot: Age (in years) by Group.

\section{Results}

3.1. Participants. Of the 255 questionnaires returned, the 136 (53\%) that contained descriptions of falls beyond home are included in the analysis presented here. In Table 1 and Figures 1 and 2, we summarise the characteristics of these 86 men and 50 women (aged from 54 to 91 years). We received responses from 19 single fallers, 86 repeat fallers (median 2 falls beyond home in a year), and 31 very frequent fallers (median 6 falls beyond home in a year).

3.2. Completeness of Data. Participants described the location of 249 falls in varying detail. In 240/249 descriptions (96\%), we discerned the activity during which they fell, and, in 226 descriptions (91\%), fallers proposed a cause. It was apparent how the faller landed in 216 descriptions (87\%). Responses to "Then what happened?" often contained more than one type of answer, so we counted comments about recovery to standing (173 descriptions, 69\%), injury (100, 40\%), and immediate healthcare (40,16\%) separately, meaning that the total number of descriptions exceeded 249. Of the 100 comments about injury, 16 stated that the fall had not caused any injury; we thus collated 84 descriptions of physical or psychological injury. Some participants had a proxy write for them or typed a response. Three comments were illegible.

3.3. Circumstances of Falling. As shown in Table 2, 38\% of falls occurred in streets or car parks and 35\% in (or at the entrance of) unfamiliar buildings, commonly shops. The proportion of 
TABLE 1: Characteristics of participants.

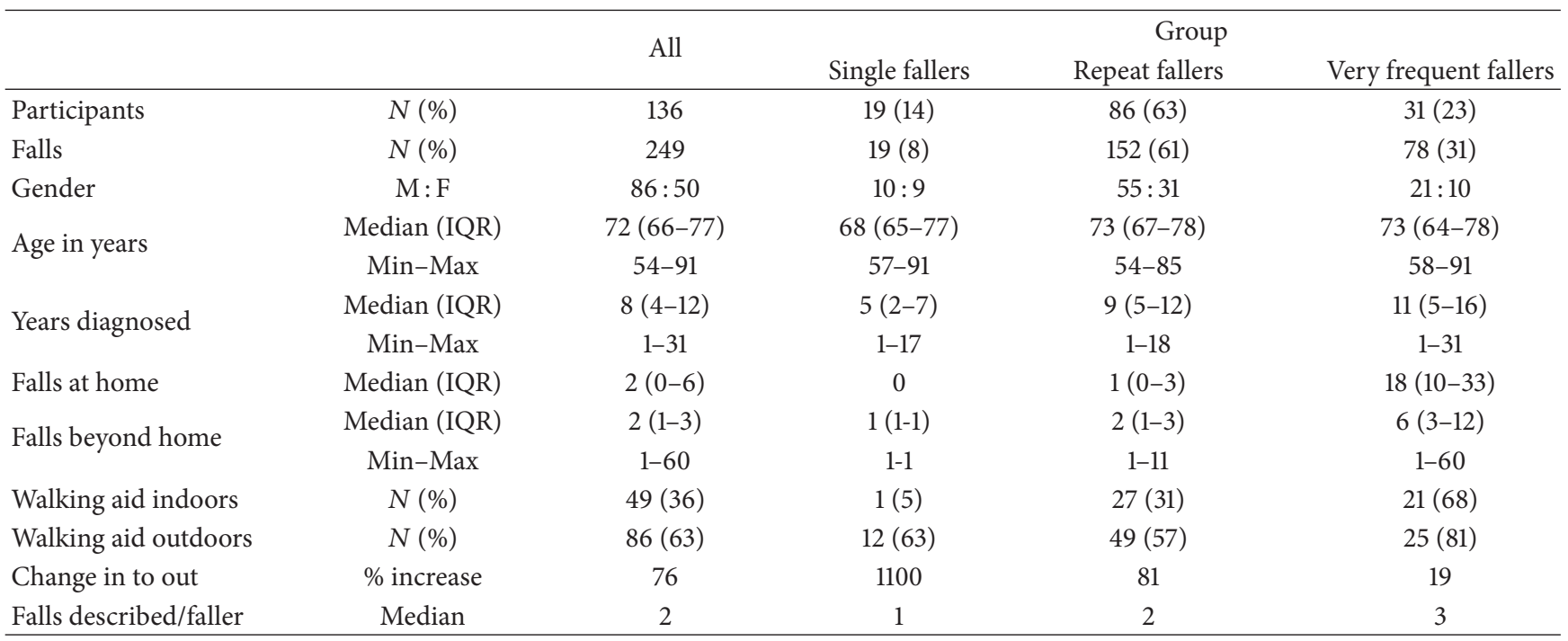

$N$ : number; \%: percent; M : F: male to females; IQR: interquartile range.

TABLE 2: Falls beyond home: locations and fall-related activity $(N=249)$.

\begin{tabular}{|c|c|c|c|c|c|}
\hline & & \multirow{2}{*}{ All } & \multicolumn{3}{|c|}{ Group } \\
\hline & & & Single fallers & Repeat fallers & Very frequent fallers \\
\hline Falls $(N)$ & & 249 & 19 & 152 & 78 \\
\hline \multirow{5}{*}{ Location } & Street/car park & $95(38 \%)$ & $8(42 \%)$ & $58(38 \%)$ & $29(37 \%)$ \\
\hline & Buildings & $85(34 \%)$ & $6(32 \%)$ & $47(31 \%)$ & $32(41 \%)$ \\
\hline & Green spaces & $59(24 \%)$ & $5(26 \%)$ & $39(26 \%)$ & $15(19 \%)$ \\
\hline & In transit & $10(4 \%)$ & 0 & $8(5 \%)$ & $2(3 \%)$ \\
\hline & Total & 249 & 19 & 152 & 78 \\
\hline \multirow{8}{*}{ Activity } & Walking & $129(52 \%)$ & $11(58 \%)$ & $79(52 \%)$ & $39(50 \%)$ \\
\hline & Strenuous (including shopping) & $44(18 \%)$ & $4(21 \%)$ & $28(18 \%)$ & $12(15 \%)$ \\
\hline & Vehicle transfers & $22(9 \%)$ & $1(5 \%)$ & $13(9 \%)$ & $8(10 \%)$ \\
\hline & On steps & $16(6 \%)$ & $1(5 \%)$ & $12(8 \%)$ & $4(5 \%)$ \\
\hline & Standing & $12(5 \%)$ & 0 & $7(5 \%)$ & $5(6 \%)$ \\
\hline & Sit or stand transfers & $12(5 \%)$ & $2(11 \%)$ & $5(3 \%)$ & $5(6 \%)$ \\
\hline & Toileting/dressing & $5(2 \%)$ & 0 & $3(2 \%)$ & $2(3 \%)$ \\
\hline & Total & $240(96 \%)$ & $19(100 \%)$ & $146(96 \%)$ & $75(96 \%)$ \\
\hline
\end{tabular}

$N$ : number, \%: percent.

"green" falls was 13\% in the countryside and 11\% in unfamiliar gardens.

Over half the falls (52\%) occurred during walking. Other effortful ("strenuous") activities, gardening, shopping, and playing with grandchildren, for example, accounted for $18 \%$ of falls. Attempting to enter or exit a vehicle (termed "vehicle transfers" in the table), predominantly cars, accounted for $9 \%$ of falls. Ascending and descending steps contributed equally to $6 \%$. Falls from standing accounted for $5 \%$ of falls, as did standing from sitting or sitting from standing (sit or stand transfers in the table).

Sudden causes of falling (like tripping) outweighed the failure to complete an action (like turning), see Table 3. Among sudden falls, tripping was most common, accounting for $24 \%$. Inadequate concentration or vigilance, attributed to distraction or fatigue, accounted for a further $12 \%$, followed in frequency by freezing (6\%). Among the failures to complete an action, fallers referred to "loss of balance" in $18 \%$ of cases and turning in 9\%. Causes grouped under "collapse" included "blood pressure," "blacked out," "legs buckled," and "stimulator failed."

Falling forward was most common, for example, on hands and knees (50\%), with almost equal numbers of backward and sideways falls (18\% each).

3.4. Immediate Consequences of Falls. The participants' reports of injury, recovery to standing and health service intervention following falls are summarised in Table 4. Participants mentioned minor injuries in $26 \%$ of descriptions and major injuries in 3\%; they specified being uninjured in 
TABLE 3: Falls beyond home: causes and landings $(N=249)$.

\begin{tabular}{|c|c|c|c|c|c|}
\hline & & \multirow{2}{*}{ All } & \multicolumn{3}{|c|}{ Group } \\
\hline & & & Single fallers & Repeat fallers & Very frequent fallers \\
\hline Falls $(N)$ & & 249 & 19 & 152 & 78 \\
\hline \multirow{7}{*}{ Cause: sudden } & Tripped & $60(24 \%)$ & $9(47 \%)$ & $37(24 \%)$ & $14(18 \%)$ \\
\hline & Distracted or tired & $29(12 \%)$ & $1(5 \%)$ & $17(11 \%)$ & $11(14 \%)$ \\
\hline & Freezing & $16(6 \%)$ & 0 & $10(7 \%)$ & $6(8 \%)$ \\
\hline & Slipped & $10(4 \%)$ & 0 & $10(7 \%)$ & 0 \\
\hline & Missed footing & $9(4 \%)$ & $2(11 \%)$ & $6(4 \%)$ & $1(1 \%)$ \\
\hline & "Collapse" & $9(4 \%)$ & $1(5 \%)$ & $1(1 \%)$ & $7(9 \%)$ \\
\hline & Total & $133(53 \%)$ & $13(68 \%)$ & $81(53 \%)$ & $39(50 \%)$ \\
\hline \multirow{7}{*}{ Cause: failure } & Lost balance & $45(18 \%)$ & $3(16 \%)$ & $30(20 \%)$ & $12(15 \%)$ \\
\hline & Turned (including too fast) & $23(9 \%)$ & $2(11 \%)$ & $14(9 \%)$ & $7(9 \%)$ \\
\hline & Rushing & $10(4 \%)$ & 0 & $7(5 \%)$ & $3(4 \%)$ \\
\hline & Dodging someone & $7(3 \%)$ & 0 & $4(3 \%)$ & $3(4 \%)$ \\
\hline & Reaching or bending & $5(2 \%)$ & 0 & $4(3 \%)$ & $1(1 \%)$ \\
\hline & Step back/small space & $3(1 \%)$ & 0 & $1(1 \%)$ & $2(3 \%)$ \\
\hline & Total & $93(37 \%)$ & $5(26 \%)$ & $60(39 \%)$ & $28(36 \%)$ \\
\hline \multirow{3}{*}{ Landing } & Forward & $125(50 \%)$ & $14(74 \%)$ & $81(53 \%)$ & $30(38 \%)$ \\
\hline & Backwards/sideways & $91(37 \%)$ & $3(16 \%)$ & $54(36 \%)$ & $34(44 \%)$ \\
\hline & Total & $216(87 \%)$ & $17(89 \%)$ & $135(89 \%)$ & $64(82 \%)$ \\
\hline
\end{tabular}

$N$ : number, \%: percent.

TABLE 4: Immediate consequences of falls beyond home $(N=249)$.

\begin{tabular}{|c|c|c|c|c|c|}
\hline & & \multirow{2}{*}{ All } & \multicolumn{3}{|c|}{ Group } \\
\hline & & & Single & Repeat & Very frequent \\
\hline Falls $(N)$ & & 249 & 19 & 152 & 78 \\
\hline \multirow{6}{*}{ Injury } & Minor injuries & $45(18 \%)$ & $5(26 \%)$ & $32(21 \%)$ & $8(10 \%)$ \\
\hline & Head or facial injury & $19(8 \%)$ & $2(11 \%)$ & $16(11 \%)$ & $1(1 \%)$ \\
\hline & No injury* & $16(6 \%)$ & $2(11 \%)$ & $8(5 \%)$ & $6(8 \%)$ \\
\hline & Shaken or embarrassed & $12(5 \%)$ & 0 & $12(8 \%)$ & 0 \\
\hline & Fracture/dislocation & $8(3 \%)$ & $1(5 \%)$ & $6(4 \%)$ & $1(1 \%)$ \\
\hline & Total & $100(40 \%)$ & $10(53 \%)$ & $74(49 \%)$ & $16(21 \%)$ \\
\hline \multirow{4}{*}{ Recovery } & Stood alone & $75(30 \%)$ & $7(37 \%)$ & $40(26 \%)$ & $28(36 \%)$ \\
\hline & Stood with known help & $49(20 \%)$ & $1(5 \%)$ & $30(20 \%)$ & $18(23 \%)$ \\
\hline & Stood with stranger's help & $49(20 \%)$ & $5(26 \%)$ & $27(18 \%)$ & $17(22 \%)$ \\
\hline & Total & $173(69 \%)$ & $13(68 \%)$ & $97(64 \%)$ & $63(81 \%)$ \\
\hline \multirow{5}{*}{ Input } & Paramedics attended & $15(6 \%)$ & $1(5 \%)$ & $9(6 \%)$ & $5(6 \%)$ \\
\hline & Attended A and E & $16(6 \%)$ & $2(11 \%)$ & $13(9 \%)$ & $1(1 \%)$ \\
\hline & Seen by doctor or nurse & $7(3 \%)$ & 0 & $7(5 \%)$ & 0 \\
\hline & Admitted to hospital & $2(1 \%)$ & 0 & $2(1 \%)$ & 0 \\
\hline & Total & $40(16 \%)$ & $3(16 \%)$ & $31(20 \%)$ & $6(8 \%)$ \\
\hline
\end{tabular}

$N$ : number, \%: percent, ${ }^{*}$ signifies that the comment was "no injury sustained," for example.

$6 \%$ of cases but feeling shaken or embarrassed in 5\%. Respondents mentioned receiving health service intervention after approximately one in six falls beyond home (16\%). In $30 \%$ of descriptions, fallers regained standing alone. In $20 \%$ of descriptions, someone familiar helped, most frequently their spouse, and, in $20 \%$, fallers mentioned someone unknown, like a passerby, helping. Single fallers mostly recovered alone, but if they received help, it was mostly unfamiliar.
Repeat- and very frequent fallers tended to receive help usually from someone they knew.

3.5. Group Specific Features. The single faller group was of youngest median age and shortest median disease duration with similar numbers of men and women. The $1100 \%$ increase among those using a walking aid indoors and out was 


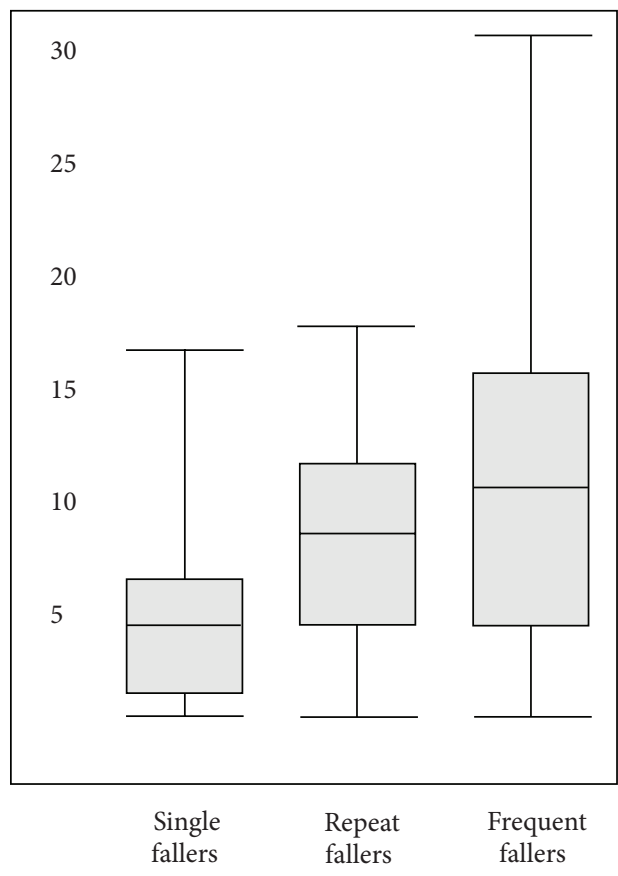

Boxes represent median and interquartile range; whiskers represent minimum and maximum values

FIGURE 2: Box and whisker plot: time since diagnosis (in years) by group.

marked. This was the group most likely to report falling while walking or changing between sitting and standing, to mention minor (or no) injury and to report attending the Emergency Department. They were relatively likely to fall after tripping or missing their footing but relatively unlikely to attribute falling to distraction or fatigue. They were more likely than other groups to fall forwards (and less likely to fall sideways or backwards). They were relatively more likely to regain standing independently or with a stranger's help after falling (and less likely to receive help from someone familiar).

The repeat fallers were the only participants to attribute falls to slipping, to report feeling shaken or embarrassed afterwards, to report seeing a doctor or nurse other than in the Emergency Department after falling, or to require hospital admission.

There were twice as many men as women among the very frequent fallers, who had the longest median disease duration and highest use of walking aids. They were more likely than other groups to fall backwards or sideways, in an unfamiliar building, after a collapse, and less likely to fall forwards, after tripping, in a green space. They were also relatively less likely to mention sustaining injury.

\section{Discussion}

Response rate and data quality were satisfactory: we received replies to approximately two-thirds of the questionnaires distributed, from PwP diagnosed between 1 and 31 years, and had to disregard only three illegible answers. We had to look widely to capture 200-plus detailed descriptions of falls: while over half our respondents had fallen at least once beyond home, most falls occur at home. However few home environments pose the challenge of maintaining postural stability while attempting complex and/or strenuous movements, on changeable underfoot conditions, amidst much noise and congestion.

Ashburn et al. reported that $80 \%$ of the falls they documented happened at home, in a study of similar sample size and participant age and disease duration as this study [3]. Prior to falling, their participants were ambulant (45\%), standing (32\%) and transferring (21\%): they attributed $11 \%$ of falls to "freezing, festination and retropulsion." In this study, we attributed a higher percentage of falls to "walking" and much smaller percentages to standing and transferring between sitting and standing. The differences in fall-related activities probably reflect differences in time use at and beyond home [13]. As we attributed only $6 \%$ of falls to freezing, it may be that starting to walk, passing through doorways, and turning round occupy proportionately less time outside the home than they do at home. There is evidence that abrupt movements frequently cause falls among PwP [14]: our respondents described falling trying to dodge people coming toward them or to step backward out of someone's way. PwP may be safer standing their ground and letting others dodge them.

Tripping after unwanted contact with the ground or other hazard dominated the causes of falling in this study and others $[3,15]$. Streets are dense with trip hazards: doorways, kerbs, steps, street furniture, raised markings to guide people with visual impairments, and random, uneven paving slabs. Grass, sand, earth, and gravel contain natural variations and obscured obstacles (like tree roots) that similarly challenge ground clearance, particularly if an individual is fatigued or distracted. Ineffective foot clearance may reflect the "deteriorating and hypokinetic motor control" underlying many falls [16].

Standing up from the ground, likely to be necessary after falling outdoors (in the absence of seating), challenges strength, flexibility, and balance. Fewer respondents described injuries than mentioned needing help to stand up. We observed potentially important differences in the proportion of our subgroups falling backward or sideways, which may follow the loss of intersegmental flexibility (or "stiffness") [17]. Falling in any direction other than forwards exposes the greater trochanter to direct trauma on landing with a high risk of significant injury $[18,19]$. Interestingly, in the current study, only 10/78 (13\%) of the very frequent fallers' descriptions of falling mentioned sustaining any physical injury, despite nearly half being described as backward or sideways. It may be that limiting respondents to describing a maximum three falls deterred them from reporting their most serious falls or that those who fall very frequently "learn" to land safely. Both suggestions require further research.

Using an appropriate walking aid outdoors may afford extra postural stability and prevent falls: (only one single faller used an aid indoors, while 12 took one outside), but devices blocking forward motion and occupying both hands may contemporaneously increase the chance of a backward 
landing if stability is lost. We found the single fallers least likely to fall backward or sideways and the very frequent fallers the least likely to fall forwards, a difference possibly attributable to greater stiffness among the latter or to their greater use of mobility aids.

Besides the injuries sustained when falling backward, these falls may be particularly embarrassing. Falling into the unknown, sustaining injury, and/or being publically embarrassed can leave the faller "shaken" or otherwise distressed. Repeat fallers were the only group to report being "shaken" by falling. Occasional falls in "embarrassing" circumstances (backwards in public and needing help to get up) may be more emotionally disturbing than frequent falls of which PwP may have learned to expect and manage or a single fall from which they are quickly back on their feet. The "shock" of a rare fall may lead some individuals (not just frequent fallers) to exhibit a fear of falling [20]. Those without a history of falls are more likely to leave home unaccompanied and are perhaps more likely to find strangers offering help if they should fall, like the single fallers in the current study. Teaching PwP how best to direct a "helper" after a fall could be as useful as teaching them how to get up alone.

PwP use their attention (and other cues) to generate and maintain movement. They seem most at risk of falls when they are distracted and fail to focus on movement. One similarity between our findings and those of Ashburn et al. [3] is in the $12 \%$ of falls they attributed to "misjudgement and distraction" and the $15 \%$ we attributed to "distraction" and "missed footing." Even among the single fallers in the current study, tripping, distraction, and missed footing accounted for 12 of the 13 falls with a sudden cause (92\%). No strategy will be universally applicable, but our finding that over $40 \%$ of the falls reported were attributed to tripping, distraction, missed footing, or rushing suggests that hypervigilance may be an effective and widely applicable fall-prevention strategy for $\mathrm{PwP}$. For $\mathrm{PwP}$ and significant cognitive impairment, hypervigilance may be a strategy more effectively employed by an accompanying carer.

Some argue that exercise aimed at increasing muscle strength, postural stability, and joint flexibility, coupled with learning new strategies for tackling challenging movements (like turns and transfers), is an effective way of reducing falls among PwP [21]. An exercise-based intervention is not universally applicable (nor desirable) [22, 23], and the supporting evidence is not strong [24]. In a recent review and meta-analysis, falls have not been among the list of outcomes for which physiotherapy was found to benefit people with Parkinson's [24]. A recent trial in which $67 \mathrm{PwP}$ exercised at home for six weeks and 67 served as controls has demonstrated no significant difference in the rates of falling at eight weeks or six months though near misses reduced significantly among those who exercised [21]. Li et al. stated that "no clinical trial has shown the efficacy of exercise in reducing falls" among PwP but went on to generate evidence for a reduction in falls after Tai Chi [25]. We agree that there is some evidence to support both exercise and the learning of new strategies (e.g., dual-task training) to improve mobility: "fall rates may underestimate positive effects of exercise" [26] if they are not adjusted for activity level. However, it is worth considering also training $\mathrm{PwP}$ to focus on one activity at a time, given the difficulties that $\mathrm{PwP}$ experience when multitasking. Learning to focus on mobility and avoid distractions surely deserves a place among the growing raft of strategies we advocate for safe mobility. PwP will need to take care to avoid trips, survive freezing episodes, and turn safely (for example) no matter how fit they are.

Despite considerable research, improved medical management of PD, and heightened awareness of the potential benefits of exercise, fall frequency among PwP shows little evidence of decreasing. We are not the first to question whether we have "oversold the benefit of late-life exercise" and to suggest that while acknowledging the current balance of evidence, we need to look beyond exercise to more "behavioural" strategies that may benefit individuals [27]. A simple change in behaviour, slowing down to focus on mobility rather than any external distractions (e.g., chatting or rushing), may effectively prevent falls and may also be faster, cheaper, and more widely applicable than some exercise-based interventions.

Effective exercise "contributes to both fitness and fatigue" [28]. Exercise-induced fatigue can impede postural control even in healthy young subjects [29]: even local muscle fatigue in the ankle dorsiflexors may diminish ground clearance. Among PwP, high background muscle tone may exacerbate physical fatigue, and the need to concentrate for long periods, applying attention to once "automatic" movements, may exacerbate mental fatigue. Thus, anyone using hypervigilance to the environment (and his or her mobility therein) as a fall avoidance strategy must couple it with a sensitivity to fatigue. If they do not rest when fatigued, physically or mentally, people may make errors of judgement [30], which could lead to falls.

We call this change from the promotion of exercise and environmental modification toward hypervigilance and fatigue management a paradigm shift because it necessitates a change in the basic assumptions made and held by the scientific community [31]. Instead of focusing on negatives, like a lack of movement speed or postural stability, we advocate focusing on excess (muscle tone, mental demand, anxiety, or fatigue, e.g.). "Slowing down and taking care" is at least as valid fall-prevention strategy as exercise and warrants further research. This study supports recent findings about PwP walking in the community, including their use of "consciously attending to walking" and "planning and preparation" as facilitating strategies and the significance of the external environment's demands as barriers [8].

This study allowed over 200 people to contribute to the study, including those with restricted mobility. Our respondents may have exhibited volunteer bias, as most were able to attend branch meetings, and being members of a support network may distinguish them from other PwP: however, data saturation was reached. The results, based on cross-sectional data supplied by respondents, could be subject to recall bias. However, in this exploratory study we did not demand total recall: we wished to know how $\mathrm{PwP}$ would describe their experiences. While respondents in any falls study may forget certain details, we are unaware of any evidence to suggest that they fabricate events. We 
have commented both on what respondents reported and where data was incomplete, which further allows the reader to assess the validity of the data. Furthermore, the subgroup analysis prevented falls by very frequent fallers dominating the picture. Future longitudinal research would illuminate the changing circumstances of falling, as some active single fallers progress to very frequent fallers over time.

\section{Conclusion}

Even independently active PwP who do not fall at home do fall in the environment beyond home (often repeatedly). Unseen or unsuccessfully negotiated physical hazards (like uneven pavements and tight parking spaces) provide frequent challenges, compounded by psychological pressures (like multitasking or rushing).

Falls alter in type as they become more frequent. Hard, backward landings, which are difficult to control and from which it is difficult to recover without help replace trips in the street, from which the uninjured faller can recover to their feet independently: rescue, assessment, and treatment by the health services become more likely. Anyone falling alone and outside is likely to be observed and assisted by someone unfamiliar. A stranger's intervention in a public fall may heighten the immediate embarrassment and have disabling consequences.

Avoiding falls in the environment beyond home requires vigilance and preparedness. Slowing down and concentrating on a single task (without unnecessary distraction) might reduce falls more effectively than waiting for the effects of an exercise programme to afford some protection and/or for pavements to be better maintained: testing this hypothesis warrants further research.

\section{Acknowledgments}

Parkinson's UK funded this study as part of a larger project: Promoting Activity and Preventing Activity-Related Falls in PD. The authors would like to thank the Parkinson's UK branches and support groups throughout Southern England who allowed us to distribute questionnaires or provided feedback on the study findings.

\section{References}

[1] G. K. Wenning, G. Ebersbach, M. Verny et al., "Progression of falls in postmortem-confirmed Parkinsonian disorders," Movement Disorders, vol. 14, no. 6, pp. 947-950, 1999.

[2] B. H. Wood, J. A. Bilclough, A. Bowron, and R. W. Walker, "Incidence and prediction of falls in Parkinson's disease: a prospective multidisciplinary study," Journal of Neurology Neurosurgery and Psychiatry, vol. 72, no. 6, pp. 721-725, 2002.

[3] A. Ashburn, E. Stack, C. Ballinger, L. Fazakarley, and C. Fitton, "The circumstances of falls among people with Parkinson's disease and the use of Falls Diaries to facilitate reporting," Disability and Rehabilitation, vol. 30, no. 16, pp. 1205-1212, 2008.

[4] D. Jones and J. Playfer, "Parkinson's Disease," in Physical Management for Neurological Conditions, M. Stokes and E. Stack, Eds., Chapter 6, Elsevier, Oxford, UK, 3rd edition, 2011.
[5] Y. P. T. Kamsma, W. H. Brouwer, and J. P. W. F. Lakke, “Training of compensational strategies for impaired gross motor skills in Parkinson's disease," Physiotherapy Theory and Practice, vol. 11, no. 4, pp. 209-229, 1995.

[6] M. E. Morris, "Movement disorders in people with parkinson disease: a model for physical therapy," Physical Therapy, vol. 80, no. 6, pp. 578-597, 2000.

[7] A. Nieuwboer, L. Rochester, and D. Jones, "Cueing gait and gaitrelated mobility in patients with Parkinson's disease: developing a therapeutic method based on the international classification of functioning, disability, and health," Topics in Geriatric Rehabilitation, vol. 24, no. 2, pp. 151-165, 2008.

[8] R. M. Lamont, M. E. Morris, H. Woollacott, and S. G. Brauer, "Community walking in people with Parkinson's disease," Parkinson's Disease, Article ID 856237, 8 pages, 2012.

[9] D. Jones, L. Rochester, A. Birleson et al., "Everyday walking with Parkinson's disease: understanding personal challenges and strategies," Disability and Rehabilitation, vol. 30, no. 16, pp. 1213-1221, 2008.

[10] E. Stack and A. Ashburn, "Dysfunctional turning in Parkinson's disease," Disability and Rehabilitation, vol. 30, no. 16, pp. 12221229, 2008.

[11] H. Stolze, S. Klebe, C. Baecker et al., "Prevalence of Gait disorders in hospitalized neurological patients," Movement Disorders, vol. 20, no. 1, pp. 89-94, 2005.

[12] W. Li, T. H. M. Keegan, B. Sternfeld, S. Sidney, C. P. Quesenberry Jr., and J. L. Kelsey, "Outdoor falls among middle-aged and older adults: a neglected public health problem," American Journal of Public Health, vol. 96, no. 7, pp. 1192-1200, 2006.

[13] M. Granat, "Can we quantify behaviour of the older adult from the analysis of free-living activities?" Journal of Aging and Physical Activity, vol. 20, p. S43, Abstracts for the 8th World Congress on Aging and Physical Activity, 2012.

[14] M. D. Willemsen, Y. A. M. Grimbergen, M. Slabbekoorn, and B. R. Bloem, "Falling in Parkinson disease: more often due to postural instability than to environmental factors," Nederlands Tijdschrift voor Geneeskunde, vol. 144, no. 48, pp. 2309-2314, 2000.

[15] E. Stack and A. Ashburn, "Fall events described by people with Parkinson's disease: implications for clinical interviewing and the research agenda," Physiotherapy Research International, vol. 4, no. 3, pp. 190-200, 1999.

[16] B. Galna, S. Lord, and L. Rochester, "Does ineffective foot clearance when walking distinguish fallers from non-fallers in people with Parkinson's disease?" Movement Disorders, vol. 27, supplement 1, article 814, 2012.

[17] Y. A. M. Grimbergen, M. Munneke, and B. R. Bloem, "Falls in Parkinson's disease," Current Opinion in Neurology, vol. 17, no. 4, pp. 405-415, 2004.

[18] S. R. Cummings and M. C. Nevitt, "A hypothesis: the causes of hip fractures," Journals of Gerontology, vol. 44, no. 4, pp. M107M111, 1989.

[19] O. Johnell, L. J. Melton, E. J. Atkinson, W. M. O'Fallon, and L. T. Kurland, "Fracture risk in patients with Parkinsonism: a population-based study in Olmsted County, Minnesota," Age and Ageing, vol. 21, no. 1, pp. 32-38, 1992.

[20] M. H. Nilsson, G.-M. Hariz, S. Iwarsson, and P. Hagell, "Walking ability is a major contributor to fear of falling in people with Parkinson's disease: implications for rehabilitation," Parkinson's Disease, vol. 2012, Article ID 713236, 7 pages, 2012. 
[21] A. Ashburn, L. Fazakarley, C. Ballinger, R. Pickering, L. D. McLellan, and C. Fitton, "A randomised controlled trial of a home based exercise programme to reduce the risk of falling among people with Parkinson's disease," Journal of Neurology, Neurosurgery and Psychiatry, vol. 78, no. 7, pp. 678-684, 2007.

[22] C. H. Whitehead, R. Wundke, and M. Crotty, "Attitudes to falls and injury prevention: what are the barriers to implementing falls prevention strategies?" Clinical Rehabilitation, vol. 20, no. 6, pp. 536-542, 2006.

[23] L. Yardley, S. Kirby, Y. Ben-Shlomo, R. Gilbert, S. Whitehead, and C. Todd, "How likely are older people to take up different falls prevention activities?" Preventive Medicine, vol. 47, no. 5, pp. 554-558, 2008.

[24] C. L. Tomlinson, S. Patel, C. Meek et al., "Physiotherapy intervention in Parkinson's disease: systematic review and metaanalysis," British Medical Journal, vol. 345, article e5004, 2012.

[25] F. Li, P. Harmer, K. Fitzgerald et al., "Tai chi and postural stability in patients with Parkinson's disease," The New England Journal of Medicine, vol. 366, pp. 511-519, 2012.

[26] L. Z. Rubenstein, K. R. Josephson, P. R. Trueblood et al., "Effects of a group exercise program on strength, mobility, and falls among fall-prone elderly men," Journals of Gerontology A, vol. 55, no. 6, pp. M317-M321, 2000.

[27] J. J. Keysor and A. M. Jette, "Have we oversold the benefit of late-life exercise?" Journals of Gerontology A, vol. 56, no. 7, pp. M412-M423, 2001.

[28] J. R. Fitz-Clarke, R. H. Morton, and E. W. Banister, "Optimizing athletic performance by influence curves," Journal of Applied Physiology, vol. 71, no. 3, pp. 1151-1158, 1991.

[29] G. C. Gauchard, P. Gangloff, A. Vouriot, J. P. Mallié, and P. P. Perrin, "Effects of exercise-induced fatigue with and without hydration on static postural control in adult human subjects," International Journal of Neuroscience, vol. 112, no. 10, pp. 11911206, 2002.

[30] R. F. Baumeister and J. Tierney, Willpower: Rediscovering the Greatest Human Strength, The Penguin Press, New York, NY, USA, 2011.

[31] T. Kuhn, The Structure of Scientific Revolutions (1962), University of Chicago Press, Chicago, Ill, USA, 1962. 


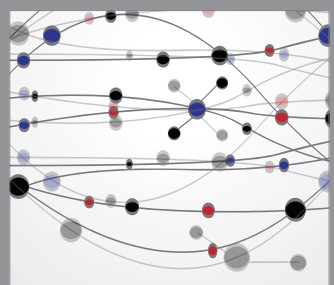

The Scientific World Journal
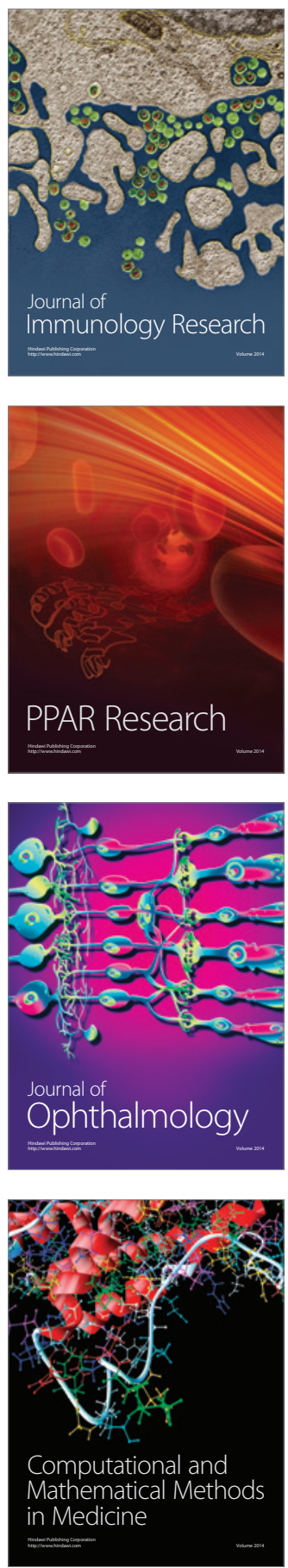

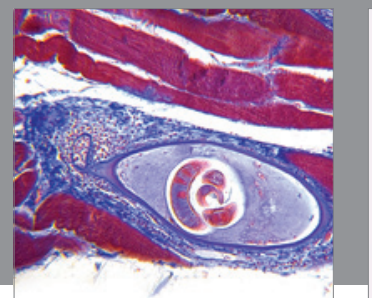

Gastroenterology

Research and Practice
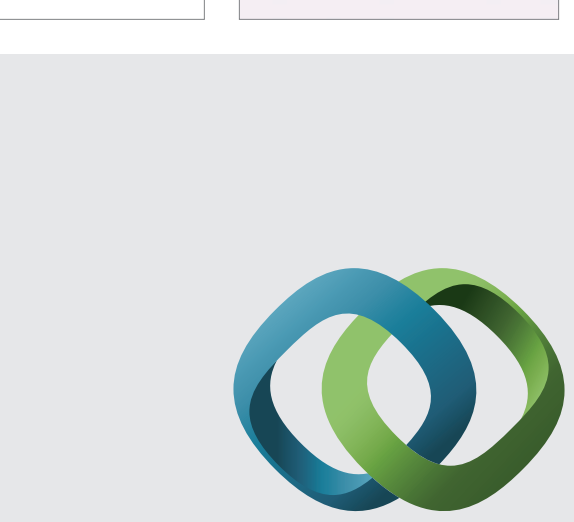

\section{Hindawi}

Submit your manuscripts at

http://www.hindawi.com
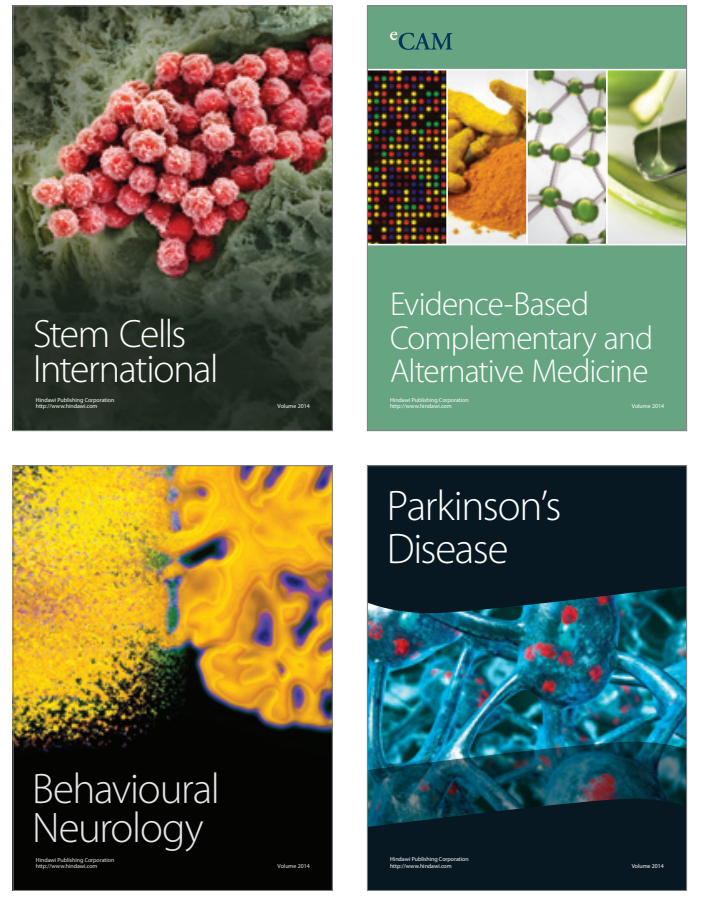
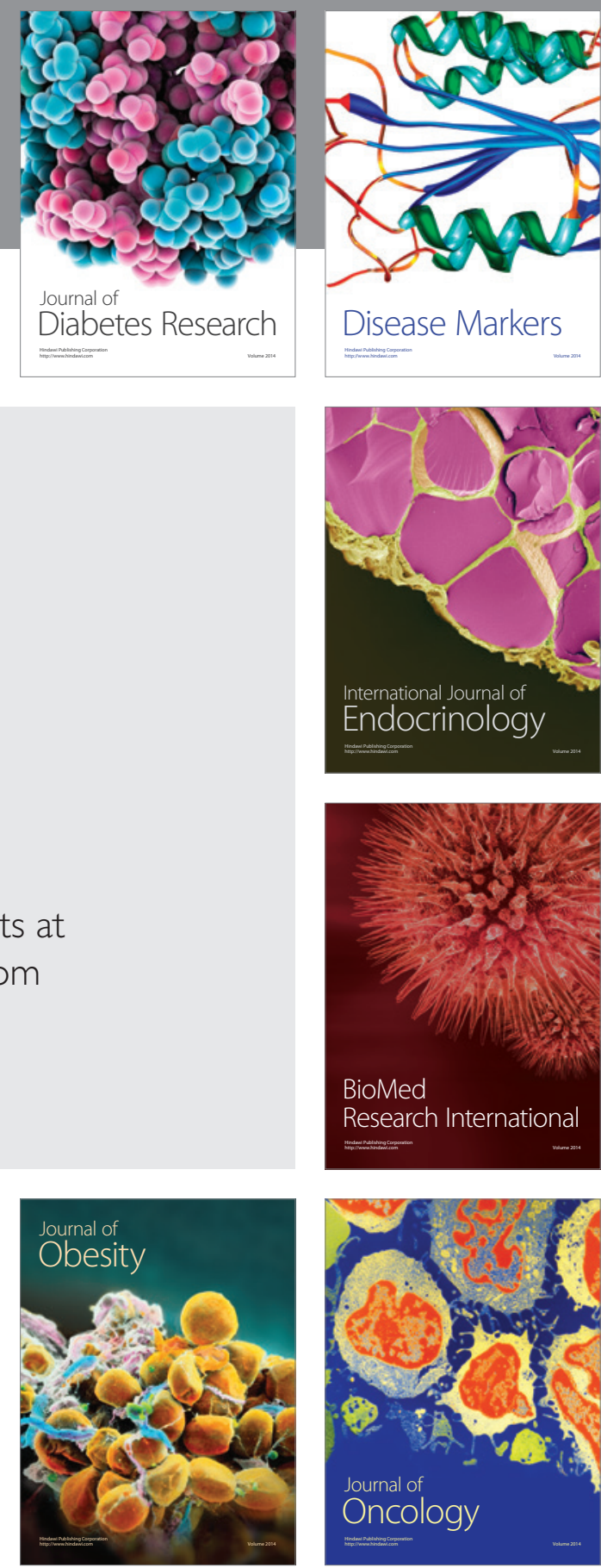

Disease Markers
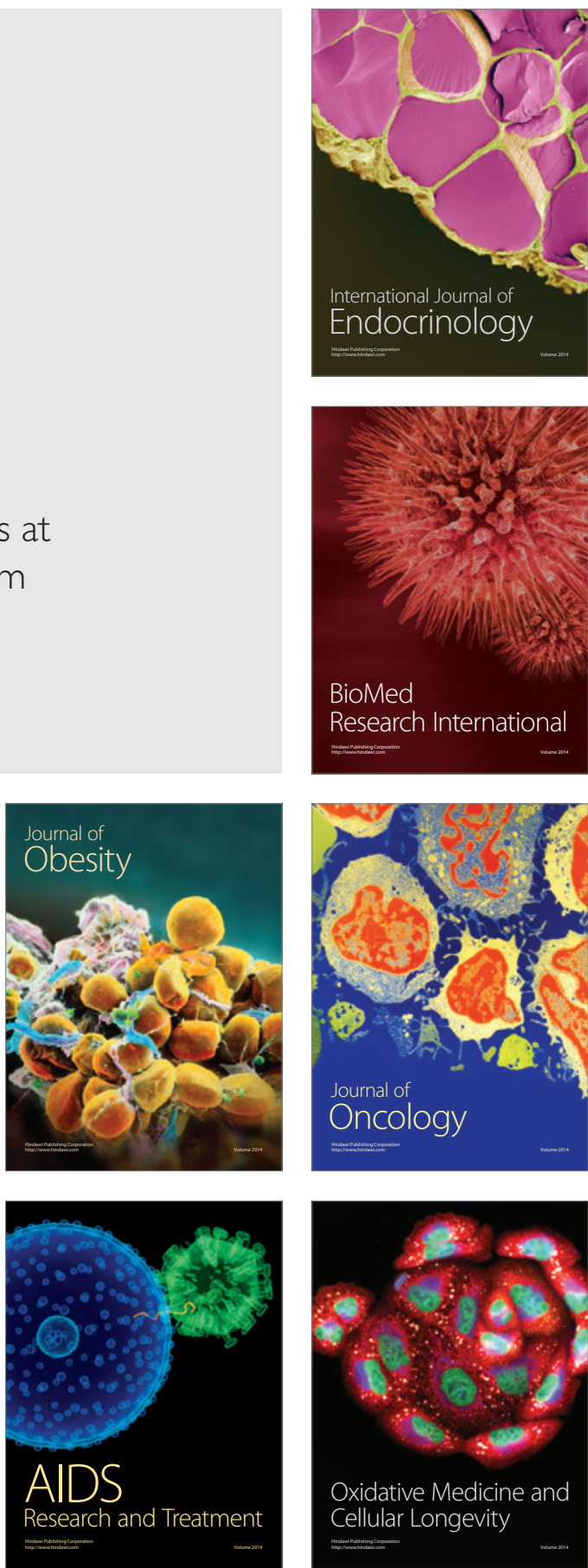